\title{
Crossover Junction Endonuclease MUS81
}

National Cancer Institute

\section{Source}

National Cancer Institute. Crossover Junction Endonuclease MUS81. NCI Thesaurus.

Code C116617.

Crossover junction endonuclease MUS81 (551 aa, $61 \mathrm{kDa}$ ) is encoded by the human MUS81 gene. This protein plays a role in the catabolism of branched DNA structures. 